Document downloaded from:

http://hdl.handle.net/10251/64126

This paper must be cited as:

Vikingsson, LKA.; Gómez-Tejedor, JA.; Gallego Ferrer, G.; Gómez Ribelles, JL. (2015). An experimental fatigue study of a porous scaffold for the regeneration of articular cartilage. Journal of Biomechanics. 48(7):1310-1317. doi:10.1016/j.jbiomech.2015.02.013.

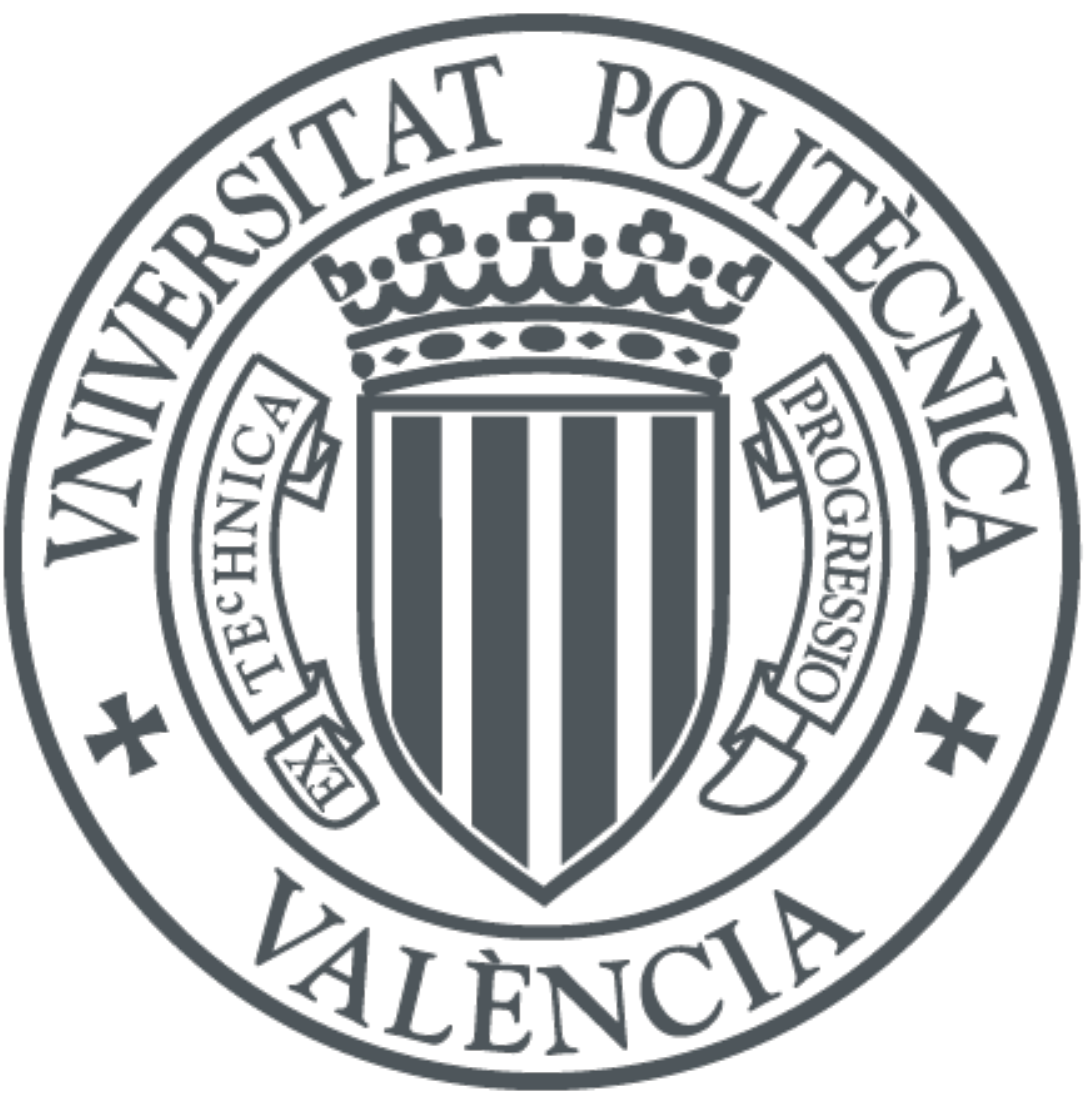

The final publication is available at

http://dx.doi.org/10.1016/j.jbiomech.2015.02.013

Copyright Elsevier

Additional Information 


\section{An experimental fatigue study of a porous scaffold for the regeneration of articular cartilage}

L. Vikingsson ${ }^{1, *}$, J.A. Gómez-Tejedor ${ }^{1}$, G. Gallego Ferrer ${ }^{1,2}$, J.L. Gómez Ribelles ${ }^{1,2}$

Journal of Biomechanics 48 (2015) 1310-1317

\section{http://dx.doi.org/10.1016/j.jbiomech.2015.02.013}

${ }^{1}$ Centre for Biomaterials and Tissue Engineering (CBIT), Universitat Politècnica de València, Camino de vera s/n, 46022 Valencia, Spain.

${ }^{2}$ Biomedical Research Networking centre in Bioengineering, Biomaterials and Nanomedicine (CIBER-BBN), Valencia, Spain.

*Corresponding author. Tel.: +34 963877007, ext. 88939; fax: +34 963877276. E-mail: livi1@upv.es

\section{Abstract}

The aim of this experimental study is to predict the long-term mechanical behavior of a porous scaffold implanted in a cartilage defect for tissue engineering purpose. Fatigue studies were performed by up to 100,000 unconfined compression cycles in a polycaprolactone $(P C L)$ scaffold with highly interconnected pores architecture. The scaffold compliance, stress-strain response and hysteresis energy have been measured after different number of fatigue cycles, while the morphology has been observed by scanning electron microscopy at the same fatigue times. To simulate the growing tissue in the scaffold/tissue construct, the scaffold was filled with an aqueous solution of polyvinyl alcohol (PVA) and subjected to repeating cycles of freezing and thawing that increase the hydrogel stiffness. Fatigue studies show that the mechanical loading provokes failure of the dry scaffold at a smaller number of deformation cycles than when it is immersed in water, and also that 100,000 compressive dynamic cycles do not affect the scaffold/gel construct. This shows the stability of the scaffold implanted in a chondral defect and gives a realistic simulation of the mechanical performance from implantation of the empty scaffold to regeneration of the new tissue inside the scaffold's pores. 


\section{Introduction}

Defects in the articular cartilage surface can lead to loss of cartilaginous tissue and osteoarthritis. Only in United Kingdom a third of the population over 45 years have sought treatment for osteoarthritis and in the United States over 27 million people are affected (Osteoarthritis in General Practice 2013). Spontaneous regeneration of articular cartilage is very limited and current clinical treatments are focused on regeneration methods by bone marrow stem cells (bMSC) through microfracture (Steadman et al. 1999), abrasion chondroplasty, drilling (Insall 1974) or mature chondrocyte implantation (Brittberg 1999). The results are promising but the tissue generated is fibro-cartilaginous with limited mechanical properties (Hunziker 2002) (Pelttari, Steck, and Richter 2008).

Hyaline cartilage is a specialized tissue containing around $75 \%$ water, and its extra cellular matrix (ECM) is composed of mainly collagen type II fibers and proteoglycan aggregates. The tissue is hard and able to sustain compression loading due to the combination of the collagen fibers stiffness and the high water sorption capacity of the glycosaminoglycans (GAGs) (Roughley and Lee 1994). When a scaffold is implanted in a cartilage defect, either seeded with chondrocytes or pluripotential cells or invaded "in vivo" by bMSC, a cartilaginous tissue is expected to grow inside the pores of the scaffold. The mechanical behavior of the implant will result from the original stiffness of the scaffold and the water permeability through the newly formed tissue. The scaffold must be able to diminish the differences in stress in relation to neighboring tissue(Hutmacher 2000) and at the same time give the cells proper mechanotransduction signals to create their ECM (Chiquet et al. 2003). The mechanical performance of the scaffold is therefore important, and due to the great influence of the newly formed tissue, "in vitro" essays of the empty scaffold are not representative for the "in vivo" situation.

Poly vinyl alcohol (PVA) aqueous solution has the interesting property to crystallize with repeated cycles of freezing and thawing (Hassan and Peppas 2000) and the resulting hydrogel is proposed by other authors as a cartilage substitute due to its high 
equilibrium water content (80-90\%), swelling capacities and good mechanical properties (Li et al. 2010)(Tamura et al. 1986). Other authors try to develop artificial cartilage combining different materials, such as PCL and alginate/polyacrylamide (Liao et al. 2013) or chitosan/agarose/gelatin (Bhat, Tripahi, and Kumar 2011) or polylactic acid and agar (Gong et al. 2007). Without doubt, the combination of synthetic or natural scaffolding materials with hydrogels for cartilage regeneration is an arising and important field. In previous studies our group filled a porous PCL scaffold with a PVA gel physically cross-linked by different cycles of freezing and thawing and evaluated its mechanical properties. It was possible to tailor the elastic modulus of the PCL-PVA construct to reach values characteristic of natural articular cartilage after six cycles of freezing and thawing (Vikingsson et al. 2014). The outcome is an cartilage model mimicking the behavior of the growing ECM inside the pores of the implanted scaffold in a cartilage defect.

In this work we have studied the long-term mechanical behavior of the PCL-PVA construct under compressive fatigue tests. The performance of the empty scaffold or filled with water has been compared to the behavior of the scaffold filled with hydrogel after six cycles of freezing and thawing, to mimic the characteristics of natural articular cartilage (Vikingsson et al. 2014). This experimental study aims to predict the mechanical performance of a macro and micro porous PCL scaffold in a cartilage defect. This is important for a better understanding of the process of surgery and implantation of the scaffold for cartilage regeneration without any animal sacrifice.

\section{Materials and methods}

$\mathrm{PCL}$, number average molecular weight $(\mathrm{Mn}) 80000 \mathrm{Da} \mathrm{Mw} / \mathrm{Mn}<2$, was obtained from Sigma Aldrich (Spain). Microspheres of Elvacite 2043 (a mixture of low molecular weight poly(ethyl methacrylate) (PEMA) and poly(methyl methacrylate) (PMMA)) with diameters ranging from 120 to $200 \mu \mathrm{m}$ were purchased from Lucite International (USA). 1,4 dioxane from Sigma Aldrich (Spain) was used as solvent for PCL, and ethanol (99\% pure) from Scharlab (Spain) was used to dissolve the dioxane in the freeze extraction process. Poly(vinyl alcohol), average molecular weight (Mw) $130000 \mathrm{Da}$, and 99+\% 
hydrolyzed was purchased from Sigma Aldrich (Spain). All the chemicals were used as received and with no further modification.

\subsection{Scaffold preparation}

The PCL scaffold was fabricated by mixing a 15 wt. \% PCL in 1,4 dioxane solution with PEMA-PMMA microspheres at a weight ratio $1: 1.25$ in a freeze extraction method to produce both macro and micro pores with a total porosity of around 90\% (RodenasRochina, Gómez Ribelles and Lebourg 2013)(Santamaría et al. 2012). The mixture was immediately frozen with liquid nitrogen, and then immersed in pre-cooled ethanol. It was kept at $-20^{\circ} \mathrm{C}$ with three changes of cold ethanol to eliminate the solvent. The scaffold was washed in ethanol at $38^{\circ} \mathrm{C}$ for 8 days with daily changes of ethanol to wash the PEMA-PMMA microspheres. The resulting scaffold was dried at room temperature under vacuum until reaching constant weight. The scaffolds were cut with circular stamps and surgical scalpels of $5 \mathrm{~mm}$ diameter and $2 \mathrm{~mm}$ high. The water immersion of the hydrophobic scaffolds was done by solvent change ethanol to water. To make sure that all the pores were filled with water the scaffolds were put in water and subjected to continuous vacuum extraction. All samples were put in water 24 hours before the PVA filling or mechanical testing.

\subsection{Hydrogel preparation}

A $10 \%$ aqueous solution of PVA was prepared by stirring at $90^{\circ} \mathrm{C}$ for 1 hour. The solution was left to cool at room temperature and then poured into custom made wells, with dimensions of $6 \mathrm{~mm}$ diameter and $4 \mathrm{~mm}$ height, and frozen for 12 hours at $-20^{\circ} \mathrm{C}$ and then thawed back to room temperature in a chamber with controlled air humidity for 8 hours. The freezing and thawing step was repeated six times. The water content in the gels was analyzed and calculated for six samples after 6 cycles of freezing and thawing and overnight immersed in water. The samples were freeze dried with $-80^{\circ} \mathrm{C}$ condensation and pressure $<100$ mbar (Lyoquest, Telstar) and the mass before and after freeze drying was measured and the difference in mass was considered to be water. A Thermo Gravimetric Analysis (TGA/StarSystem, Mettler Toledo) to $400^{\circ} \mathrm{C}$ was done to evaluate the resting amount of water in the gel. The 
Journal of Biomechanics 48 (2015) 1310-1317

http://dx.doi.org/10.1016/i.jbiomech.2015.02.013

mass loss until $180^{\circ} \mathrm{C}$ was considered to be water.

\subsection{Scaffold / hydrogel construct}

The PVA solution was introduced into the already water immersed PCL scaffold by vacuum injection by syringe. The scaffold with the gel inside the pores was freeze and thawed during the same conditions as the pure PVA gel for six cycles. The effectiveness of the PVA filling was calculated through gravimetric analysis according to equation 1, by measuring the construct volume and weighing the empty PCL scaffold and the PCL scaffold filled with PVA. The density of the PVA solution was estimated from a $10 \%$ PVA aqueous solution with a pure PVA density of $1.30 \mathrm{~g} / \mathrm{cm}^{3}$ (Hassan and Peppas 2000). The PCL density is $1.146 \mathrm{~g} / \mathrm{cm}^{3}$ (Labet and Thielemans 2009).

\subsection{Mechanical testing}

Cylindrical scaffolds of $(5.00 \pm 0.04) \mathrm{mm}$ diameter and $(2.00 \pm 0.01) \mathrm{mm}$ height were cut with circular stamps and surgical scalpels. The pure PVA hydrogels were produced in costume made wells with a diameter of $(6.00 \pm 0.05) \mathrm{mm}$ and $(4.00 \pm 0.01) \mathrm{mm}$ height. 6 samples were tested in each mechanical assay, and each sample dimension was measured three times to ensure the accuracy of the sample geometry. The empty scaffolds, the water immersed scaffolds and the scaffold and gel constructs, so as the pure PVA gels, were subjected to 100,3000, 10,000 and 100,000 cycles of $1 \mathrm{~Hz}$ sinusoidal compression of $15 \%$ strain in a Microtest Universal Fatigue machine with a $1500 \mathrm{~N}$ cell. The chosen strain of $15 \%$ ensures that the samples are subjected to a higher compressive fatigue strain than the samples would suffer inside the human body, which would be less than 6\% strain (Eckstein et al. 2005). The number of cycles has been chosen to compare short-time with long-time effects inside the scaffolds. The frequency of $1 \mathrm{~Hz}$ tends to imitate the frequency of a normal human step (Eckstein et al. 2000) After each fatigue time the samples were measured in a Termo-Mechanical Assay machine (TMA) Seiko TMA/SS6000 (Japan), with two successive programs of loading and unloading to $100 \mathrm{~g}$ with a rate of $3000 \mathrm{~g} / \mathrm{min}$ in room temperature. The experiments in the TMA machine were also made for the samples without fatigue, serving as control. 


\subsection{Scanning Electron Microscopy}

The morphology of the scaffold and gel construct was observed by Scanning Electron Microscope (JEOL JSM-5410, Japan, acceleration voltage of $15 \mathrm{kV}$, frozen at $-80^{\circ} \mathrm{C}$, sublimated during 40 minutes) equipped with a cryogenic device. The samples were cut inside the cryogenic equipment to see the cross-section of the samples and covered with gold to be observed.

\subsection{Differential Scanning Calorimetry}

Differential Scanning Calorimetry (DSC) heating scans were performed at $20^{\circ} \mathrm{C} / \mathrm{min}$ in a PYRYS-DSC 8000 equipment (Perkin Elmer) under flowing nitrogen atmosphere between $-90 \circ \mathrm{C}$ and $90 \circ \mathrm{C}$ in $20 \mu \mathrm{L}$ aluminum pans for the dry and water immersed ( 24 hours) PCL scaffolds. The excess water was absorbed by filter paper immediately before introducing the sample in the equipment.

\section{Results and discussion}

\subsection{Analysis of the water content of the PVA gels}

The average water content of the hydrogels, obtained as the difference in mass of the hydrogel after 6 cycles of freezing and thawing and 24 hours immersion in water and the dry samples after freeze drying, was $(86.73 \pm 1.85) \%$. The TGA curves show a mass loss of $(4.12 \pm 0.29) \%$ up to $180^{\circ} \mathrm{C}$ that was assigned to the remaining water, In this way the PVA hydrogels contain $(86.73 \pm 1.85) \%$ and $(4.12 \pm 0.29) \%$ water.

\subsection{Morphology}

Before mechanical testing the dry PCL scaffold has micro, $(10 \pm 5) \mu \mathrm{m}$, and macro, (180 \pm 60$) \mu m$ pores (Figure 1a). These mean pores size were obtained by measuring the size of 10 micro and macro pores in 3 different SEM photos. The structure is similar to the ones obtained in previous works (Santamaría et al. 2012) (Lebourg, Suay Antón, and Gómez Ribelles 2010) (Deplaine et al. 2013) (Lebourg et al. 2013). Without fatigue, the structure of the dry and immersed scaffold is very similar. Not until 10,000 cycles of fatigue a change is seen (Figure $1 \mathrm{f}-1 \mathrm{~g}$ ), between the dry and immersed samples. The dry scaffold is clearly affected by the dynamic loading applied, the pores are collapsed and the overall scaffold structure is changed in comparison to earlier fatigue times 
(Figure 1f). For the immersed scaffold the fatigue effect is not that comprehensive (Figure 1g), the scaffold trabeculae are not broken but a compressive effect exerted by the load is noticed, as the pores become more oval shaped. The dry scaffold after 100,000 cycles of fatigue (Figure $1 \mathrm{~h}$ ) is now almost totally collapsed and many trabeculae are broken, see the arrow in Figure $1 \mathrm{~h}$. In the immersed samples after 100,000 cycles (Figure 1i) the pores are compressed but have not collapsed to the same extent as in the dry samples. The difference between the dry and immersed scaffolds shows the effect of the water inside the pores of the scaffold. The water inside the pores acts as unitary tensors with forces working against the compressive force and the applied load is not as damaging as for the samples without liquid. 


\section{Number of compression cycles}

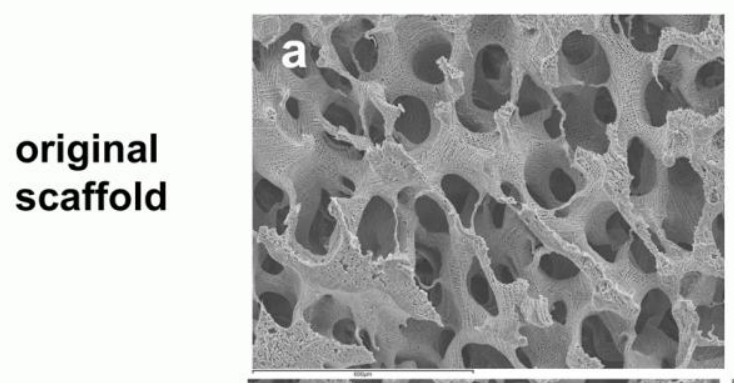

100
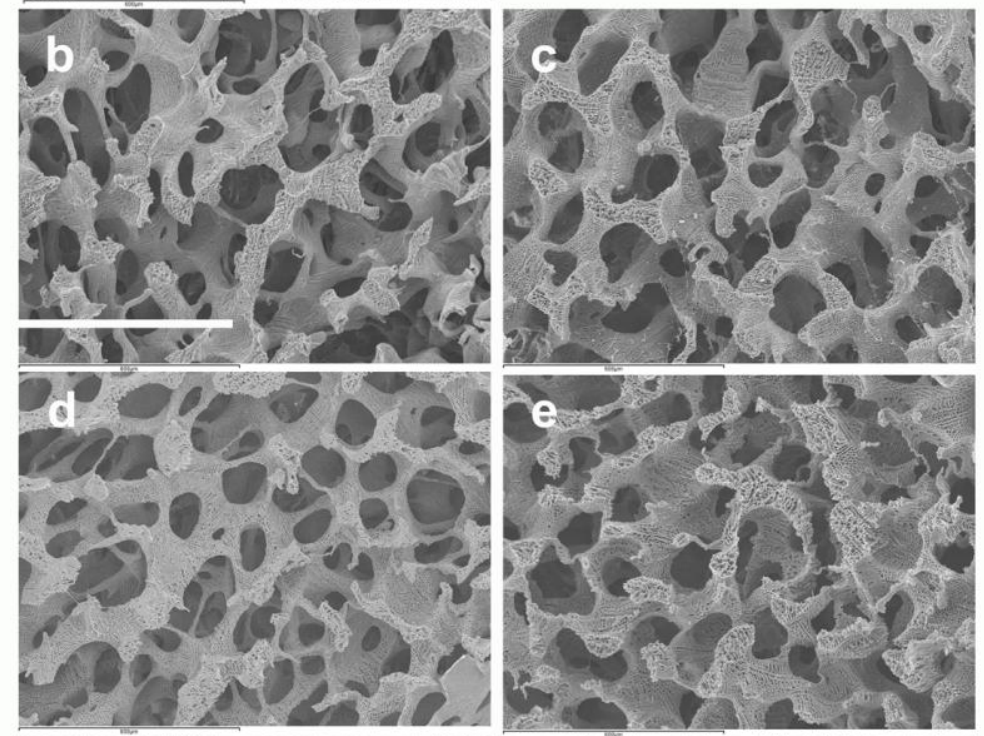

3000

10000
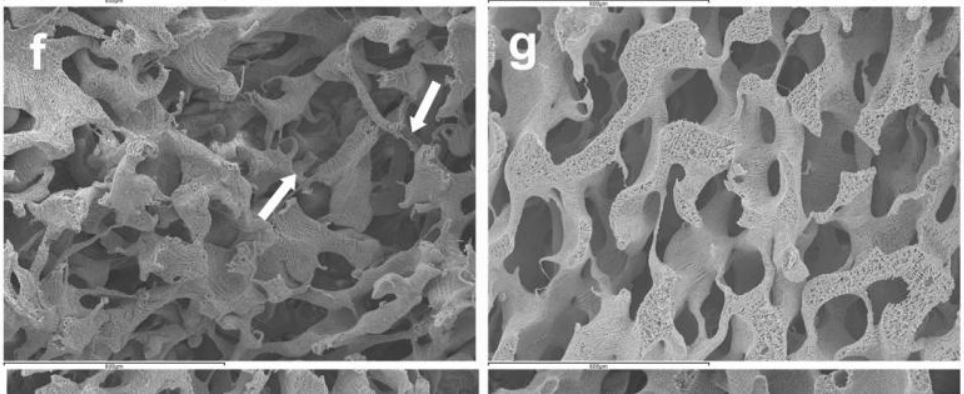

100000
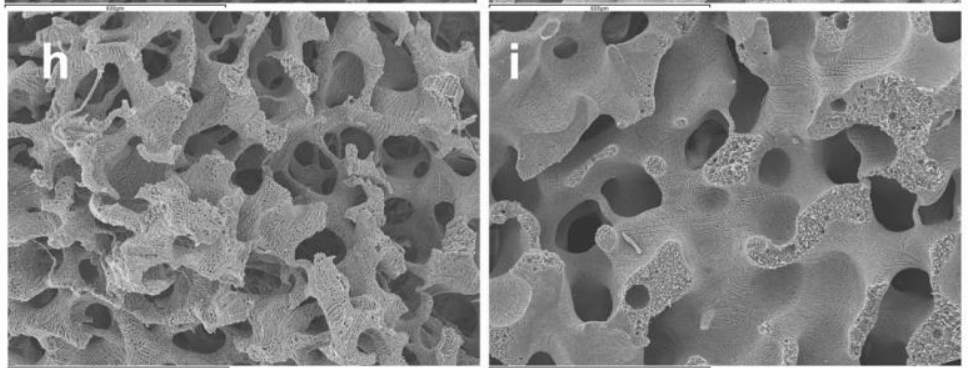

Figure 1. SEM microphotographs before (a) and after ( $b-i)$ different numbers of deformation cycles in thefatigue tests for the dry and immersed PCL scaffold. Scale bar in all figures corresponds to $600 \mu \mathrm{m}$. The arrows in the picture show some examples of broken scaffold trabeculae. 
This effect is even more emphasized for the PCL scaffold filled with PVA gel. It is worth to notice that the PVA solution enters both the micro and macro pores of the scaffold, forming a continuous phase that fills all scaffold voids. The calculated porosity according to equation 1 was zero, which probes the effectiveness of the vacuum filling of the PVA solution. No pore size change of the PCL scaffold is expected during the freezing and thawing step, the water inside the pores freezes and expands but during the thawing a retraction is expected. The porous honeycomb structure of the PVA gel is seen inside the pores of the PCL before fatigue in Figure 2a and 2c. The morphology of the PCL scaffold filled with PVA gel remains unchanged during the fatigue test (Figure 2b).

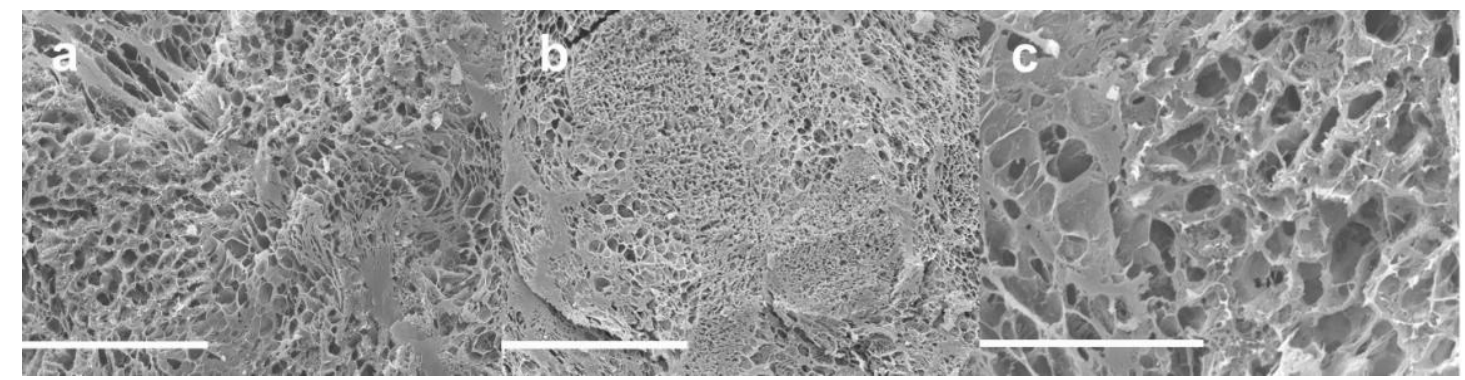

Figure 2. SEM pictures of crosssections of the PCL scaffold filled with PVA gel before (a) and after (b) 100000 cycles of fatigue (scalebar $100 \mu \mathrm{m}$ both in (a) and (b)). Figure 2c shows the bare PVA gel amplified to see the porous honeycomb structure (scalebar $40 \mu \mathrm{m}$ ).

\subsection{Differential Scanning Calorimetry}

The DSC heating scan is represented in figure 3 and shows the graph of the heat flow and temperature for the dry and water immersed $\mathrm{PCL}$ samples. The calculated crystallinity is $45 \%$ for both the samples and the melting peak is 3 degrees lower for the water immersed sample. The tg is around -60 oc for both the dry and immersed sample with a broad transition and low heat capacity increment due to the high crystallinity. A large fraction of the polymer chains that do not participate in the crystallites is immobilized in an amorphous rigid phase and hence do not participate in the glass transition. This feature seems to be enhanced by freeze extraction (Alves et al. 2002)(Wang et al. 2005)(Lebourg, Suay Antón, and Gómez Ribelles 2008). The plasticizing effect of the water adsorbed in the amorphous phase (Blasia et al. 2005). of the polymer could not be quantified This effect is considered very small comparing 
the effect of the mobility of the water inside the macro pores of the scaffold, supporting the compression loads.

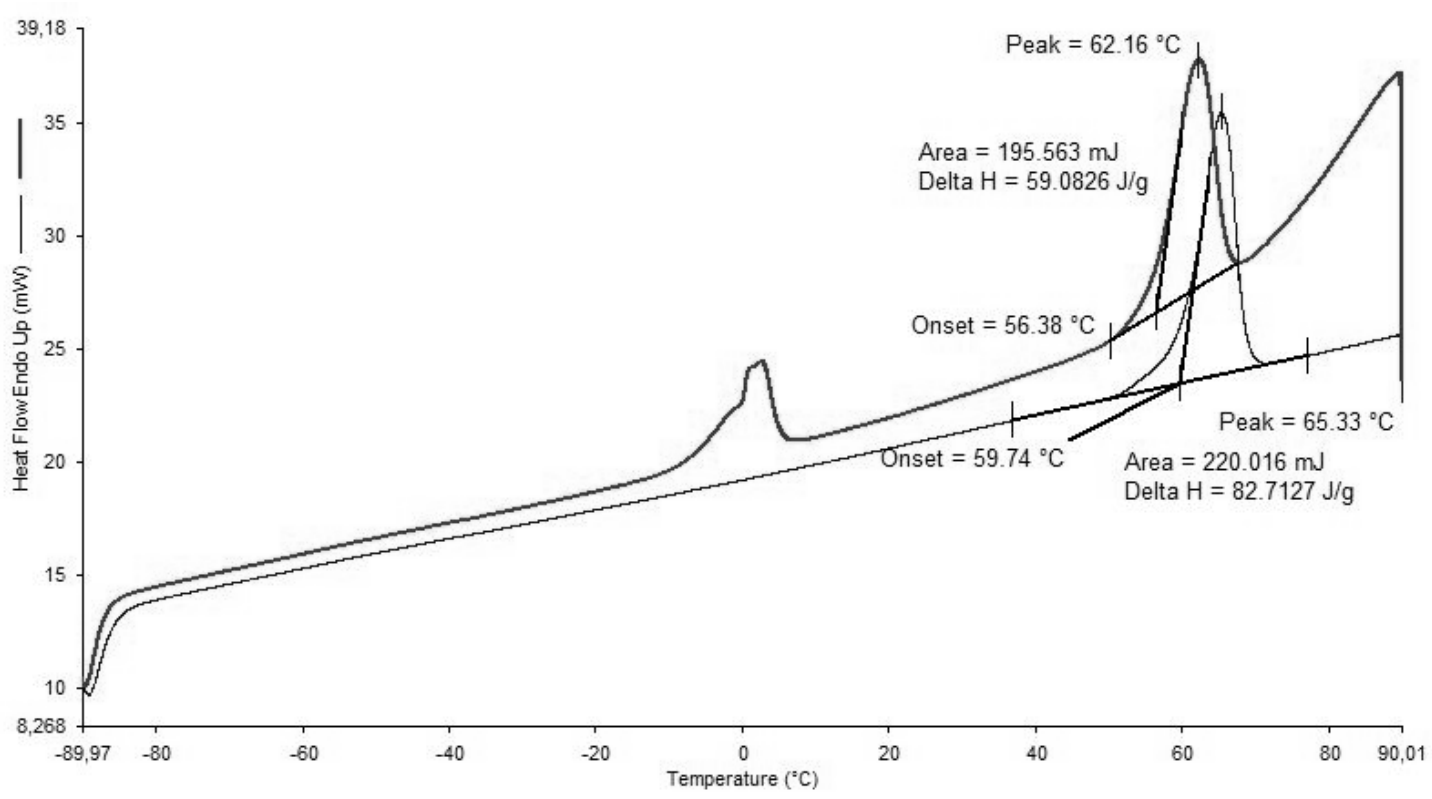

Figure 3. The DSC scan of the dry (gray line) and water immersed (black line) PCL scaffolds. The figure shows the onset, temperature peak and delta $\mathrm{H}$ for the melting peaks of the samples. The peak at $\mathrm{O}{ }^{\circ} \mathrm{C}$ for the immersed PCL represent the water evaporation from the sample. The glass transition appears around $-60 \stackrel{\circ}{\circ}$ for both samples with a broad transition and low heat capacity increment due to the high crystallinity in the PCL. A large fraction of the polymer chains that do not participate in the crystallites is immobilized in an amorphous rigid phase and hence do not participate in the glass transition.

\subsection{Mechanical properties}

\section{First and second loading curves}

The PVA gels are showing an almost perfect elastic stress strain response with loading and unloading paths that nearly overlap (Figure 4a). Furthermore no difference is seen before and after 100,000 cycles of fatigue. The dry and immersed PCL scaffolds (Figure $4 \mathrm{~b}$ and $4 \mathrm{c}$ ) show a different result, with a clear difference in the first and second loading path before fatigue. After 100,000 cycles of fatigue the curves are moved to smaller strains and the loading and unloading paths are much closer to each other indicating a more compact material where the pores have collapsed. The PCL/PVA construct shows (Figure $4 \mathrm{~d}$ ) a result combining the elasticity of the PVA gel and the stiffness of the PCL scaffold. It is seen a certain permanent deformation in the PCL scaffolds. In the PVA gel the strain is returning to its original state of zero displacement 
after unloading the charge. For the dry PCL scaffold the unloading curve reaches values of about $15 \%$ deformation and the water immersed scaffolds of about $12 \%$ deformation. This difference could be due to the water inside the pores of the scaffolds. Water is being pushed out of the scaffold pores but is also able to be reabsorbed in the cyclic compressive assays. For the PCL and PVA construct the permanent deformation is around $3 \%$.
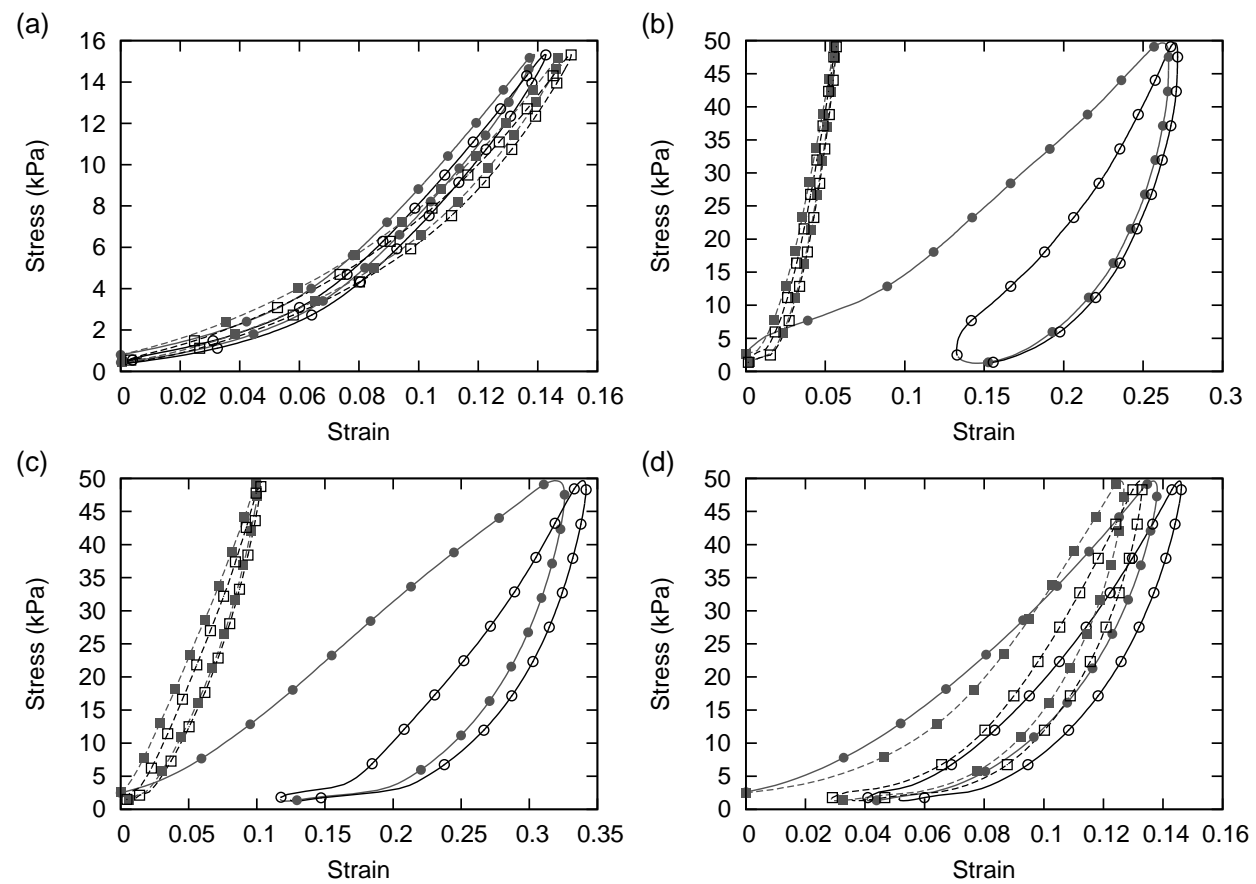

Figure 4. Stress-strain curves of the first (solid symbols) and second (open symbols) loading and unloading curves for the different samples. The solid line and circles represent the measurement before fatigue, while the curves after 100, 000 cycles of fatigue are represented by the dashed line with squares. (a) Pure PVA gel, (b) dry PCL scaffold, (c) immersed PCL scaffold and (d) the PCL/PVA construct.

\section{Second stress-strain curves}

The evolution of the compression curves for each time of fatigue (Figure 5) balance the observations from the SEM photos. It is seen how the dry and immersed samples show smaller strains after fatigue indicating a more compact material (Figure $5 b$ and $c$ ). The PCL/PVA scaffold (Figure $5 \mathrm{~d}$ ) has a less prominent strain decrease behavior, proving the effect of the PVA gel inside the pores of the scaffold. The strain for the same load 
applied is not significantly changed after any of the fatigue times for up to 100,000 cycles proving the stability of the construct.
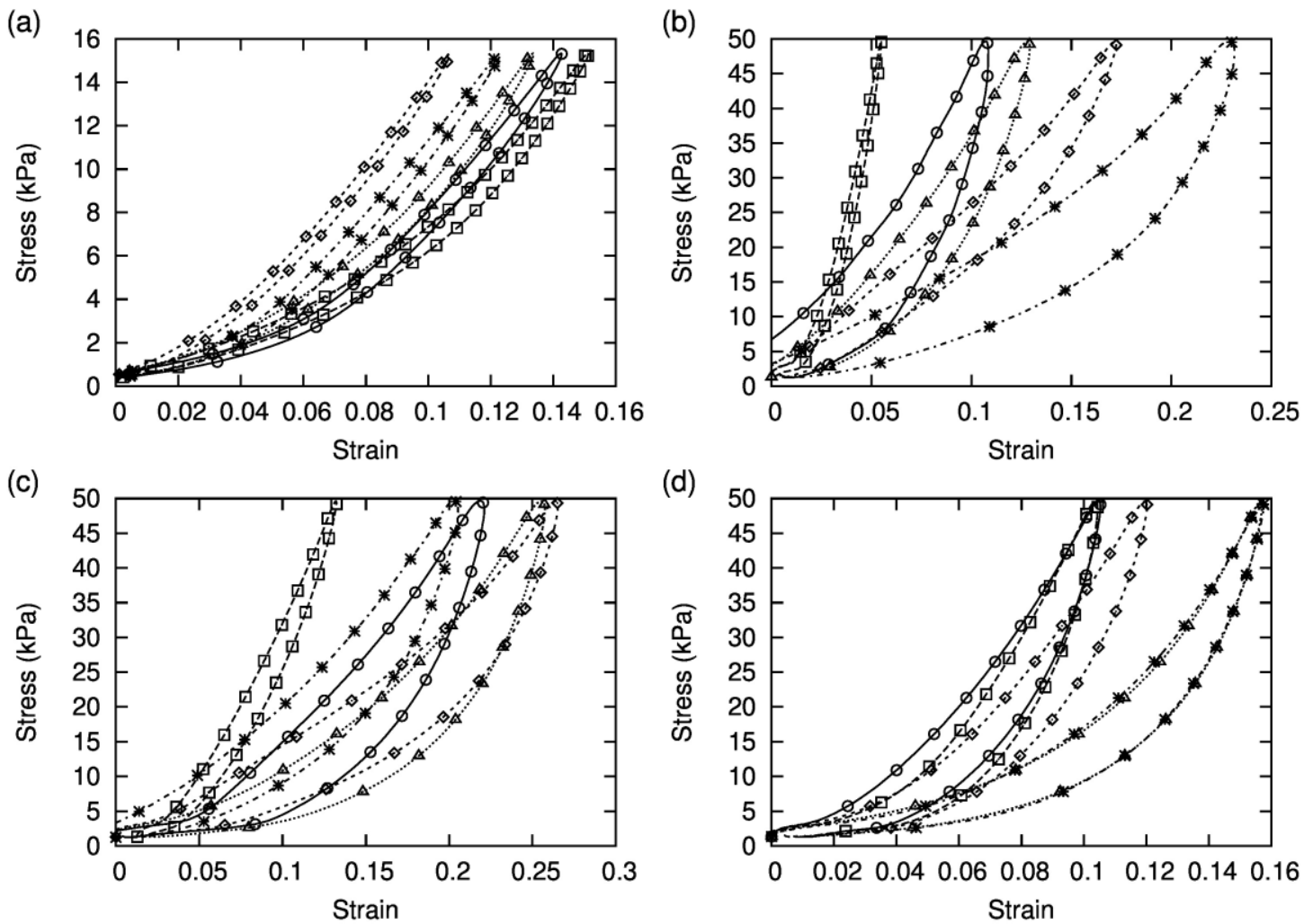

Figure 5. Stress-strain curves for the second cycle of loading for each of the times of fatigue. 0 cycles: open circles, continuous line. 100 cycles: triangles, dot line. 3000 cycles: diamonds short dashed line. 10, 000 cycles: asterisk, dot dashed line. 100, 000 cycles: open squares, long dashed line. (a) Pure PVA gel, (b) dry PCL scaffold, (c) PCL immersion and (d) PCL/PVA construct.

\section{Compliance for the first and second loading curves}

The results for the compliance the PVA gel (Figure 6a) show a quite high compliance for both the first and second loading curve for the samples without fatigue and after 100 cycles of fatigue. There is no statistical difference between the compliance for the first and second loading curves neither throughout the cycles of fatigue, except for the first loading curve at 3000 cycles, indicating stable gels not changing the mechanical behavior for up to 100,000 cycles of fatigue.

The dry PCL samples show no significant difference between the compliance of the first and second loading curves or for increasing cycles up to 10,000 cycles of fatigue (Figure $6 b$ ). The results have high standard deviation due to the porous nature of the samples. For 100,000 cycles the material is now denser, less porous and harder and 
consequently the compliance is much smaller. The pore damage is irreversible. SEM pictures in Figure 1 confirm these results.

The water immersed PCL scaffolds (Figure 6c) without fatigue show similar results as the dry samples until the the second loading curves that show the effect of water inside the pores. The compliance is increasing in the second loading curve, giving a softer material when the water is expulsed. The dry samples are hardening in the second loading curve, indicating that the material already is denser due to pore buckling. The compliance is slowly decreasing with increasing number of fatigue cycles showing that the samples are affected by the fatigue. After 100,000 cycles of fatigue the material is clearly damaged by the fatigue but in comparison to the dry samples they have been less damaged.

The compliance of the PCL scaffolds with PVA gel inside (Figure 6d) do not show any significant change during the fatigue cycles. The hydrogel inside the pores can host a great amount of water, which easily can flow within the scaffold and sustain the loads. 

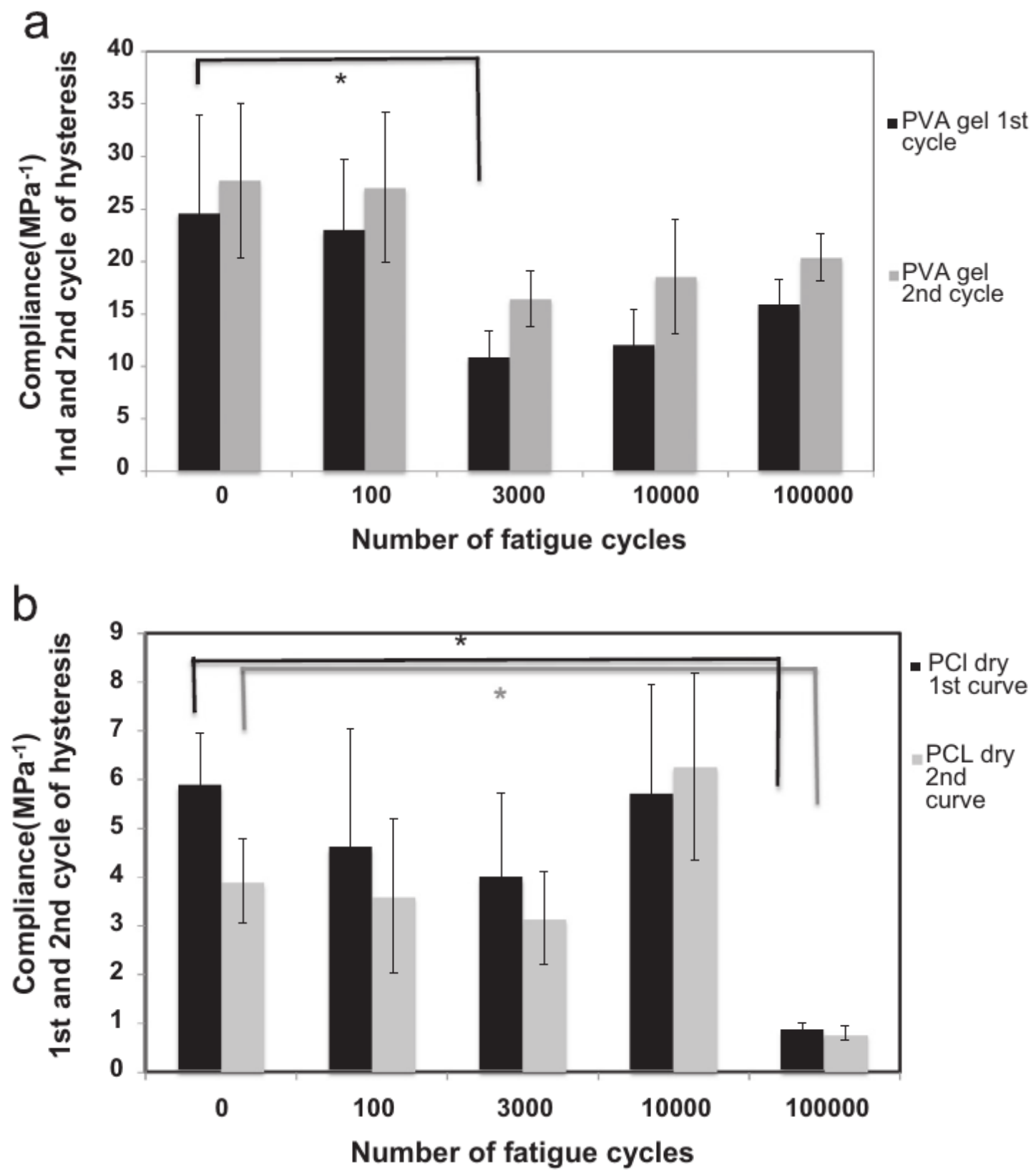

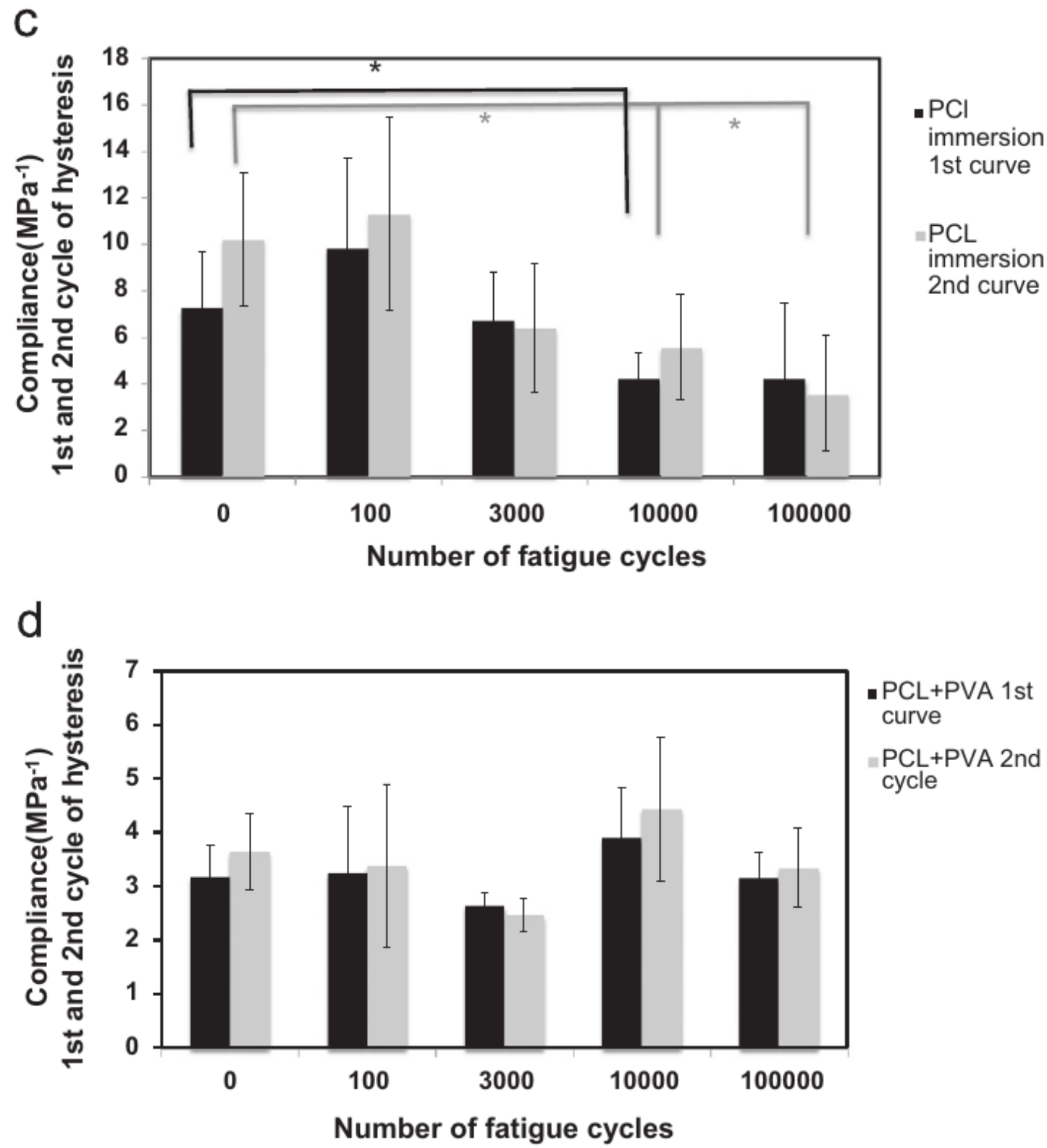

Figure 6. Compliance (mean value 7 standard deviation) for the first (black) and second (gray) loading curve for the studied systems: (a) PVA gel, (b) dry PCL, (c) immersed PCL, and PCL/PVA (d), as a function of the number of fatigue cycles. ANOVA statistical test was done, and when significant statistical difference was found an unpaired t-test was performed to evaluate the statistical difference between tested groups with $p<0.05$. Black asterisk is marked for statistical significant difference between the compliance of the first loading curves and grey asterisk for the second loading curve.

\section{Deformation at maximum load}

By observing the strain for the maximum tension applied similar results are obtained (Figure 7). The dry scaffolds have a strain around $12 \%$ for the samples without fatigue 
and there is no significant difference up to 10,000 cycles of fatigue, with just a slight increase in the maximum strain. For 10,000 cycles the strain increases to approximately $25 \%$ for the same load. For 100,000 cycles of fatigue the strain diminishes to around $5 \%$. The material should be very densely packed with little porosity and behaves as a hard material. The SEM images and the values of compliance are in good agreement with these results (Figure 1 and 6).

The water-immersed scaffolds have an initial strain of about $20 \%$ and then show a slight increase in the strain, indicating some trabeculae bending. After 100,000 cycles the strain is higher than in the dry scaffold, meaning that it has more intact pores. This is confirmed with the SEM images that show the difference of the dry and immersed scaffold after fatigue (Figure $1 \mathrm{~h}$ and $1 \mathrm{i}$ ).

The scaffold filled with PVA gel does not show any significant difference before or between the different cycles of fatigue. The strain is around $12 \%$.

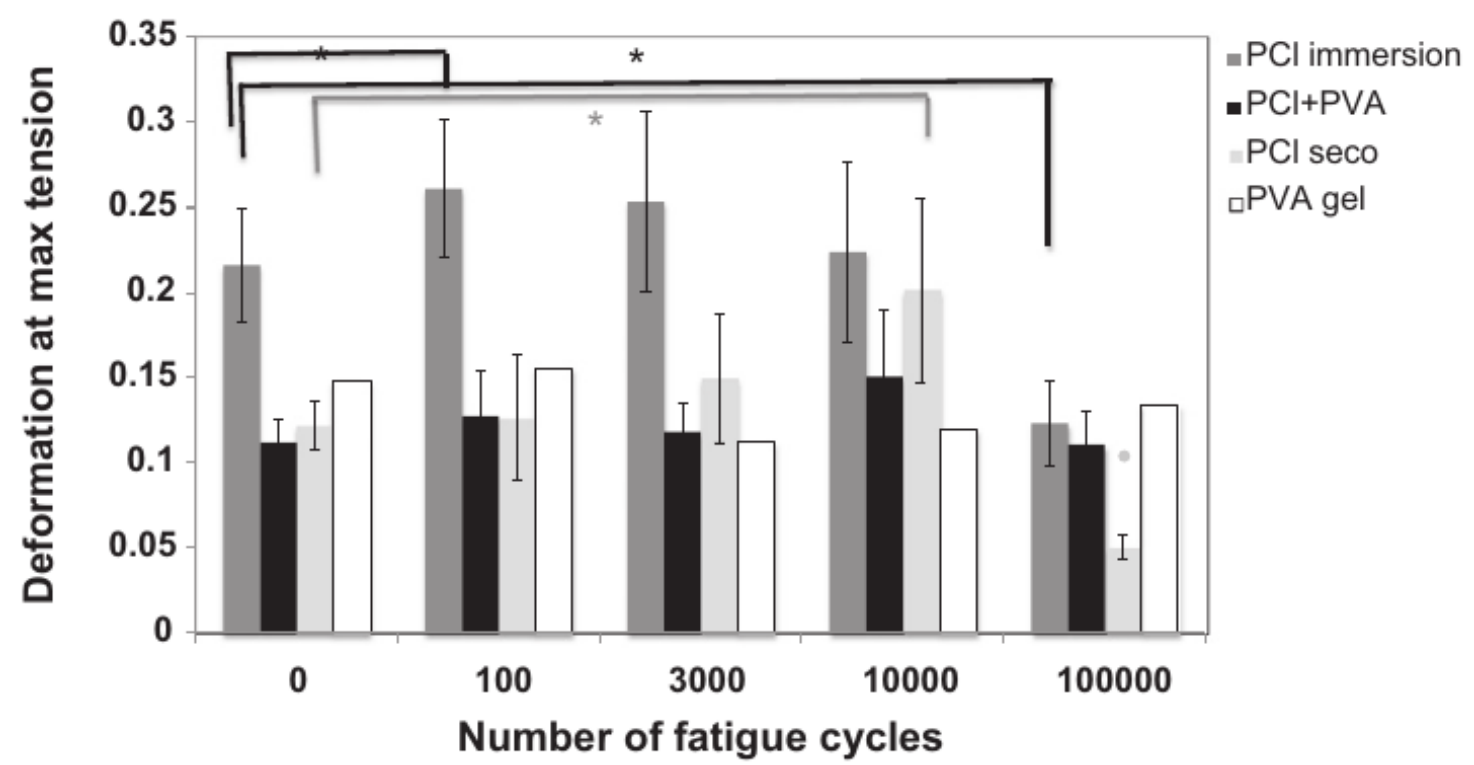

Figure 7. Deformation at maximum tension for the studied systems: PVA gel (white), dry PCL (light gray), water immersed PCL (gray), and PCL/PVA (black), as a function of the number of fatigue cycles. ANOVA statistical test was done, and when significant statistical difference was found an unpaired $\mathrm{t}$-test was performed to evaluate the statistical difference between tested groups with $\mathrm{p}<$ 0.05. Black asterisk marc statistical significant difference between PCL immersion groups and grey asterisk between PCL dry groups. 


\section{Plastic strain energy density (hysteresis energy)}

All the samples show hysteresis energy (Figure 8). A perfect elastomer does not show any hysteresis curve, the loading and unloading path are identical and no energy is dissipated (Love 1927) (Ewing 1889). In previous work (Panadero et al. 2013) a macro and micro porous PCL scaffold was filled with a fibrin gel and mechanically tested for fatigue predictions up to 1000 cycles. The hysteresis energy was calculated and with a mathematical model it was shown that filling the PCL scaffold with water or fibrin improved the fatigue life of the scaffold. In this study, for the samples without fatigue testing, the dry and immersed scaffolds have a higher plastic strain energy density compared to the scaffolds filled with hydrogel. The PCL/PVA construct shows no significant difference in the dissipated energy throughout the fatigue cycles compared to the samples without fatigue. The dry and immersed PCL scaffolds dissipate less energy after 100,000 cycles of fatigue which again shows a dense material provoked by pores collapse.

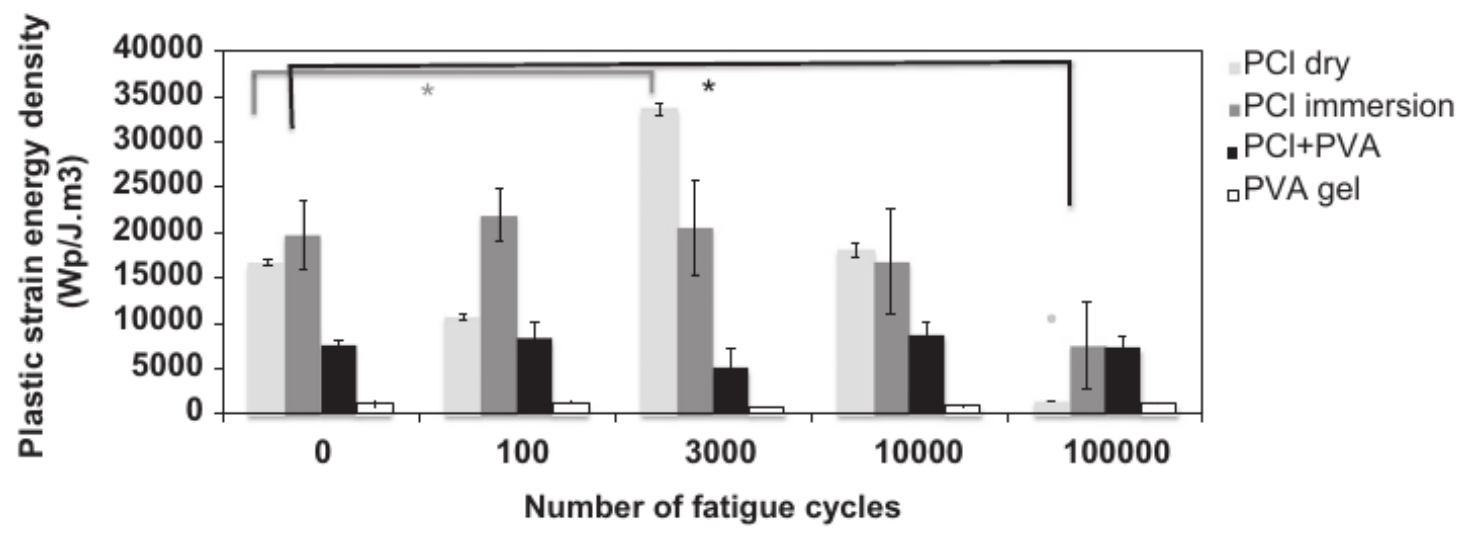

Figure 8. The dissipated energy from the second hysteresis cycle from each time of fatigue for the PVA gel (white), dry PCL (light gray), water immersed PCL (gray) and PCL/PVA construct (black). ANOVA statistical test was done, and when significant statistical difference was found an unpaired t-test was performed to evaluate the statistical difference between tested groups with $p<0.05$. Grey asterisk marc statistical significant difference between PCL dry samples and black asterisk between PCL immersion samples.

\section{Conclusions}

In this work fatigue tests have been carried out on a micro and macro porous biodegradable polycaprolactone scaffold in different conditions, dry and immersed 
state and filled with a PVA hydrogel mimicking the ECM of cartilage. Unconfined compression studies up to 100,000 cycles has been made and the compliance, maximum strain and hysteresis energy was calculated to evaluate the long -term mechanical behavior of this "in vitro" cartilage model. The first and second loading curves of the resulting stress-strain curves have been analyzed for each cycle of fatigue and the morphology has been observed after each fatigue cycle. This gives a comprehensively study of the mechanical behavior of the scaffold in implanted conditions.

The scaffold/gel construct sustains up to 100,000 cycles of fatigue without any alterations in morphology, stress-strain response or hysteresis energy. The scaffold can withstand the compressive force without trabeculae breaking since the water swollen gel inside the pores perceives some of the compressive load. This is crucial in tissue engineering applications where the scaffold is implanted and need to resist the initial physiological mechanical stress and offer an adequate biomechanical environment for the cells and the ECM. The results are important when it comes to design and fabrication of porous scaffolds to avoid unnecessary animal trials. In vivo there will be a complex interaction between the cartilage GAGs, the collagen fibers and the water molecules. Water will pass by the ECM components and go through the scaffold trabecular between the pores and to a high extent contribute to the mechanical performance. The results obtained in this study show that the scaffold is able to initially sustain the load applied, and once the cartilaginous tissue grows inside the pores the physiological load applied does not change its mechanical performance.

\section{Acknowledgements}

This work was funded by the Spanish Ministry of Economy and Competitiveness (MINECO) through the project MAT2013-46467-C4-1-R (including the FEDER financial support). CIBER-BBN is an initiative funded by the VI National R\&D\&i Plan 2008-2011, Iniciativa Ingenio 2010, Consolider Program, CIBER Actions and financed by the Instituto de Salud Carlos III with assistance from the European Regional Development 
Fund. The authors acknowledge the assistance and advice of Electron Microscopy Service of the UPV

\section{References}

Alves, N M et al. 2002. "Glass Transition and Structural Relaxation in SemiCrystalline Poly(ethylene Terephthalate). A DSC Study." Polymer 43(15): 4111-22.

Bhat, S, A Tripahi, and A Kumar. 2011. "Supermacroprous Chitosan-AgaroseGelatin Cryogels: In Vitro Characterization and in Vivo Assessment for Cartilage Tissue Engineering." J. R. Soc. Interface 8(540-554).

Blasia, P, S D’Souzab, F Selminc, and P P DeLucad. 2005. "Plasticizing Effect of Water on Poly(lactide-Co-Glycolide)." Journal of Controlled Release 108(1): 19.

Brittberg, M. 1999. "Autologous Chondrocyte Transplantation.” Clinical orthopaedics and related research 367: S147-55.

Chiquet, M, A S Renedo, F Huber, and M Flück. 2003. "How Do Fibroblasts Translate Mechanical Signals into Changes in Extracellular Matrix Production?" Matrix Biol 22(1): 73-80.

Deplaine, $\mathrm{H}$ et al. 2013. "Biomimetic Hydroxyapatite Coating on Pore Walls Improves Osteointegration of poly(L-Lactic Acid) Scaffolds." J Biomed Mater Res B Appl Biomater 101(1): 173-86.

Eckstein, F et al. 2000. "Patellar Cartilage Deformation in Vivo after Static versus Dynamic Loading." J Biomech 33(7): 819-25.

- - - 2005. "In Vivo Cartilage Deformation after Different Types of Activity and Its Dependence on Physical Training Status.” Ann Rheum Dis 64: 291-95.

Ewing, J A. 1889. On Hysteresis in the Relation of Strain to Stress. Newcastle-uponTyne.

Gong, Y et al. 2007. "Hydrogel-Filled Polylactide Porous Scaffolds for Cartilage Tissue Engineering." J Biomed Mater Res Part B: Applied Biomater 82(B): 192204.

Hassan, C M, and N A Peppas. 2000. "Structure and Applications of Poly(vinyl Alcohol) Hydrogels Produced by Conventional Crosslinking or by Freezing/thawing Methods." Advances in Polymer Science 153: 37-65. 
Hunziker, E B. 2002. "Articular Cartilage Repair: Basic Science and Clinical Progress. A Review of the Current Status and Prospects." Osteoarthritis Cartilage 10(6): 432-63.

Hutmacher, D W. 2000. "Scaffolds in Tissue Engineering Bone and Cartilage." Biomaterials 21(24): 2529-43.

Insall, J. 1974. "The Pridie Debridement Operation for Osteoarthritis of the Knee." Clin Orthop Relat Res (101): 61-67.

Labet, M, and W Thielemans. 2009. "Synthesis of Polycaprolactone: A Review." Chem. Soc. Rev. 38: 3484-3504.

Lebourg, M et al. 2013. "Different Hyaluronic Acid Morphology Modulates Primary Articular Chondrocyte Behavior in Hyaluronic Acid-Coated Polycaprolactone Scaffolds." J Biomed Mater Res A 101(2): 518-27.

Lebourg, M, J Suay Antón, and J L Gómez Ribelles. 2008. "Porous Membranes of PLLA-PCL Blend for Tissue Engineering Applications." European Polymer Journal 44(7): 2207-18.

———. 2010. "Hybrid Structure in PCL-HAp Scaffold Resulting from Biomimetic Apatite Growth." J Mater Sci Mater Med 21(1): 33-44.

Li, F, Y L Su, D F Shi, and C T Wang. 2010. "Comparison of Human Articular Cartilage and Polyvinyl Alcohol Hydrogel as Artificial Cartilage in Microstructure Analysis and Unconfined Compression." Advanced Materials Research 87: 188-93.

Liao, I-C et al. 2013. "Composite Three-Dimensional Woven Scaffolds with Interpenetrating Network Hydrogels to Create Functional Synthetic Articular Cartilage." Advanced Functional Materials 23(47): 5833-39.

Love, A E. 1927. Treatise on the Mathematical Theory of Elasticity. Dover Book. New York: Dover Publications.

Osteoarthritis in General Practice. Arthritis Research UK. 2013.

Panadero, J A et al. 2013. "Fatigue Prediction in Fibrin Poly-E-Caprolactone Macroporous Scaffolds." Journal of the Mechanical Behavior of Biomedical Materials 28(0): 55-61.

Pelttari, K, E Steck, and W Richter. 2008. “The Use of Mesenchymal Stem Cells for Chondrogenesis." Injury 39(1): 58-65.

Rodenas-Rochina, J, J L Gómez Ribelles, and M Lebourg. 2013. “Comparative Study of PCL-HAp and PCL-Bioglass Composite Scaffolds for Bone Tissue Engineering." Journal of Materials Science: Materials in Medicine 24: 12931308. 
Roughley, P J, and E R Lee. 1994. "Cartilage Proteoglycans: Structure and Potential Functions." Microsc Res Tech 28(5): 385-97.

Santamaría, V A et al. 2012. "Influence of the Macro and Micro-Porous Structure on the Mechanical Behavior of Poly (l-Lactic Acid) Scaffolds." Journal of NonCrystalline Solids 358(23): 3141-49.

Steadman, J R, W G Rodkey, K K Briggs, and J J Rodrigo. 1999. “The Microfracture Technique to Treat Full Thickness Articular Cartilage Defects of the Knee." Der Orthopäde 28(1): 26-32.

Tamura, K et al. 1986. "A New Hydrogel and Its Medical Application." Trans Amer Soc Artif Organs 32: 605.

Vikingsson, L, G Gallego Ferrer, J A Gómez-Tejedor, and J L Gómez Ribelles. 2014. "An in Vitro Experimental Model to Predict the Mechanical Behaviour of Macroporous Scaffolds Implanted in Articular Cartilage." Journal of the mechanical behavior of biomedical materials.

Wang, Y, J L Gómez Ribelles, M Salmerón Sánchez, and J F Mano. 2005.

"Morphological Contribution to Glass Transition in poly(L-Lactic Acid)." Macromolecules 38: 4712-18. 